\title{
Biomechanical Analysis of Snowboard Riding Motions
}

\author{
Chong-Hoon Lee ${ }^{1}$ and Ki-jeongNam ${ }^{2}$ and Jin-ho Back ${ }^{3 *}$ \\ ${ }^{1,2}$ Department of Sports Science Seoul National University of Science \& \\ Technology, Seoul, Korea \\ ${ }^{3}$ Dept. of Leisure Sports, Kangwon National Univ., Samcheok, Korea \\ ${ }^{1}$ leejh36@snut.ac.kr, ${ }^{2}$ rugg3@snu.ac.kr, ${ }^{3 * j h b a c k @ k a n g w o n . a c . k r ~}$ \\ *corresponding Author
}

\begin{abstract}
The present study was conducted to biomechanics data on snowboard riding in order to help the improvement of the performance of the members of the national snowboarding team. Based on the results of this study, the following conclusions can be drawn. In neutral positions, the 'up'motions were taken in which the heights of the right and left hips were relatively the highest and at the moments of turns in both directions, 'down'motions were taken in which the heights of the right and left hips were relatively the lowest. During front side turns, the right ankle angle became smaller than the left ankle angle so that the toe edge moved deeper into the snow. Muscle activity during front side turns, the right vastus intermedius muscle, the right biceps femoris muscle, the right gastrocnemius muscle, and the right anterior tibial muscle were shown to be major activated muscles. During backside turns, the right vastus lateralis muscle, the right vastus intermedius muscle, the left biceps femoris muscle, and the right anterior tibial muscle (12.64\%) were observed as major activated muscles. Plantar pressure analysis during front side turns, the largest vertical force and plantar pressure acted on the left front food and the right hind foot. During backside turns, the largest vertical force and plantar pressure acted on the left hind foot and the right hind foot. In further studies, the comparison of positions in successive turns is recommended.
\end{abstract}

\section{Introduction}

Snowboarding has become a sport popular in winter, steadily loved by youths each year. It is a highly active sport and involves very high risks of injuries when played without systematic training. Nevertheless, snowboarding has been in the limelight as a sport for pursuing harsh and free thrills in its short history (Bladin, McCrorym, \& Pogorzelski, 2004; Pressman, Johnson, 2003). Snowboarding games were first adopted as an official sport in the Olympic Games during the 1998 Nagano Winter Olympic Games. In particular, in the 2018 Pyeongchang Winter Olympic Games, a total of 10 gold medals will be awarded to men and women in five snowboarding events: parallel slalom, parallel giant slalom, snowboard cross, half-pipe, and slope styles. Since the elite snowboarding games involve high risks of injuries due to high speed, quite high jumps, and the presentation of free and new techniques, body balance, as well as muscle strength, and muscle endurance are required to prevent injuries (Pollock \& Wilmore, 1990). In addition, among many elements for judgment of the performance levels of players, the degree of development of muscle strength plays an important role in the evaluation of the performance (Oshea,1998).

Despite this importance, accurate data for sport scientific analysis methods for snow sport events cannot be easily obtained due to natural environmental constraints. For this reason, snowboarding related studies are lacking. In situations of snowboarding training on snow or games, instructors guide players with video imaging or qualitative 
evaluations. Quantitative data analysis that operates expensive equipment or utilizes sports science can prove to a difficult task. Data on qualitative analysis of snowboard riding should be helpful for guidance in the field. The present study was conducted to provide scientific data on snowboard riding in order to help the improvement of the performance of the members of the national snowboarding team.

\subsection{Study Method}

\subsection{Study Subjects}

Four national-class male elite snowboarding players were measured. All players use the regular stance (Regular-with the right foot positioned front and the left foot positioned rear) and wear a free style deck, binding, and boots. The physical characteristics of the players are given in Table 1.

Table 1. Characteristics of the Measurement Subjects (M)

\begin{tabular}{c|c|c|c}
\hline Age (year) & Height $(\mathbf{c m})$ & Weight $(\mathbf{k g})$ & Career (year) \\
\hline \hline $24.75 \pm 2.06$ & $171.75 \pm 0.96$ & $68.25 \pm 4.43$ & $4.25 \pm 0.50$ \\
\hline
\end{tabular}

\section{Experimental Tool}

The experimental equipment used in the present study is shown in Table 2.

Table 2. Detailed Information on the Experimental Equipment

\begin{tabular}{c|c|c|c}
\hline Measuring tool & Model & Quantity & Manufacturer \\
\hline Digitalcamera & DSR-PD170 & 9 & Sony \\
\hline LED & VSAD-LED & 4 & Visol \\
\hline Controlbox & & 70 & Visol \\
\hline EMG system & Telemyo 2400T & 1 & Noraxon \\
\hline Plantar pressure system & Pedar X & 1 & Novel \\
\hline
\end{tabular}

Nine digital cameras were used to conduct 3D motion analysis and the time points of individual cameras were tuned using LED. The imaging speed was set to 60fields/sec and the shutter speed was set to $1 / 200 \mathrm{sec}$. In addition, to obtain 3D coordinates of the analysis section, control boxes were used such that the entire performance section could be included.

In addition, a Noraxon electromyographic system (Telemyo 2400T system, Noraxon, USA) was used to analyze the maximum muscle strength of lower limb muscles. Bipolar surface electrodes were attached to the surfaces of the skin of the experimental subjects to collect electromyographic signals; the data was transmitted via a Wi-Fi wireless digital communication method, at a sampling rate of $1000 \mathrm{~Hz}$.

A plantar pressure sensor (Pedar X system, Novel, Germany) was used to measure plantar pressure with a sampling rateof $100 \mathrm{~Hz}$. Figure 1 shows the views of the subjects wearing the experimental equipment. 


\section{Figure 1. Views of the Subject Wearing EMG Electrodes and the Pedar Sensor}

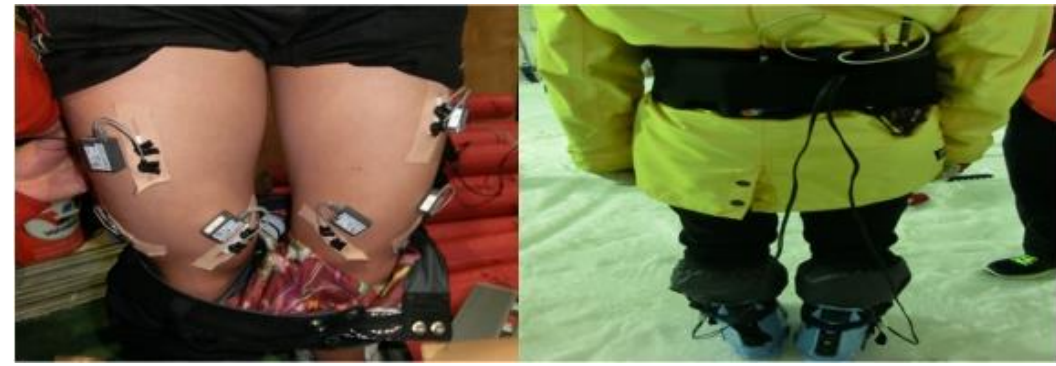

\section{Experimental Procedure}

The purpose of the present study was to calculate the kinematic variables of alpine snowboard turning motions. The experimental participants were given explanations of the experiment and signed written agreements before they participated in the experiment. All the participants performed sufficient stretching and warm-up exercises and began to prepare for the experiment when their physical conditions became sufficiently good enough for participation. The experiment was conducted in an indoor ski arena. Firstly, before conducting the experiment, control boxes were installed obliquely on the slope so that the entire analysis section could be sufficiently included and the images of the control boxes were taken. When the imaging of the control boxes was complete, the first experimental subjects began to participate in the experiment.

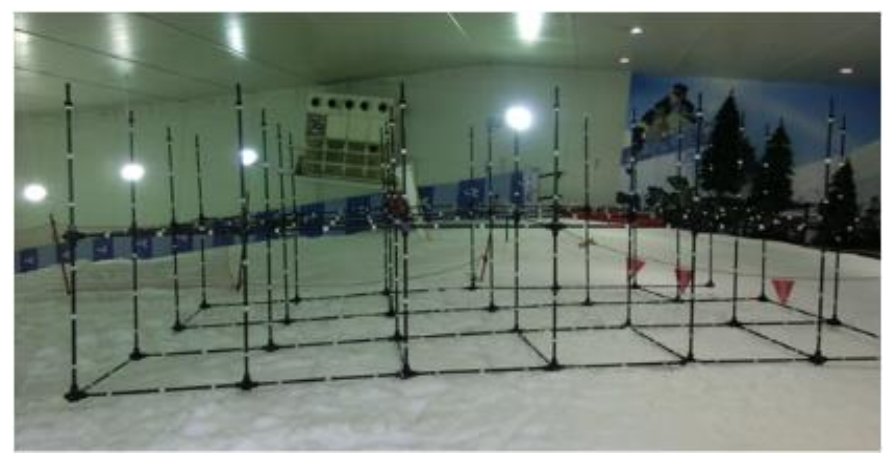

Figure 2. A View of the Control Boxes Installed

\section{Data Analysis}

Kwon 3D 3.1 is an image analysis programthat was used for the coordinates of the control boxes, the central points of human joints, and the board plate, tuning, 3D coordinate calculation using the DLT method, and data smoothing and Ms Office Excel 2010 andthe Matlab R2009bprogram were used for the calculation of other analysis variables. Before the 3D coordinate calculation, the raw data was smoothened with a 6 Hzcut-offfrequency using low-pass filters to remove the high frequency noise.

\section{1) Analysis Section Setting}

The events and phases for the experiment were set referring to a previous study(Muller et al., 1998). 


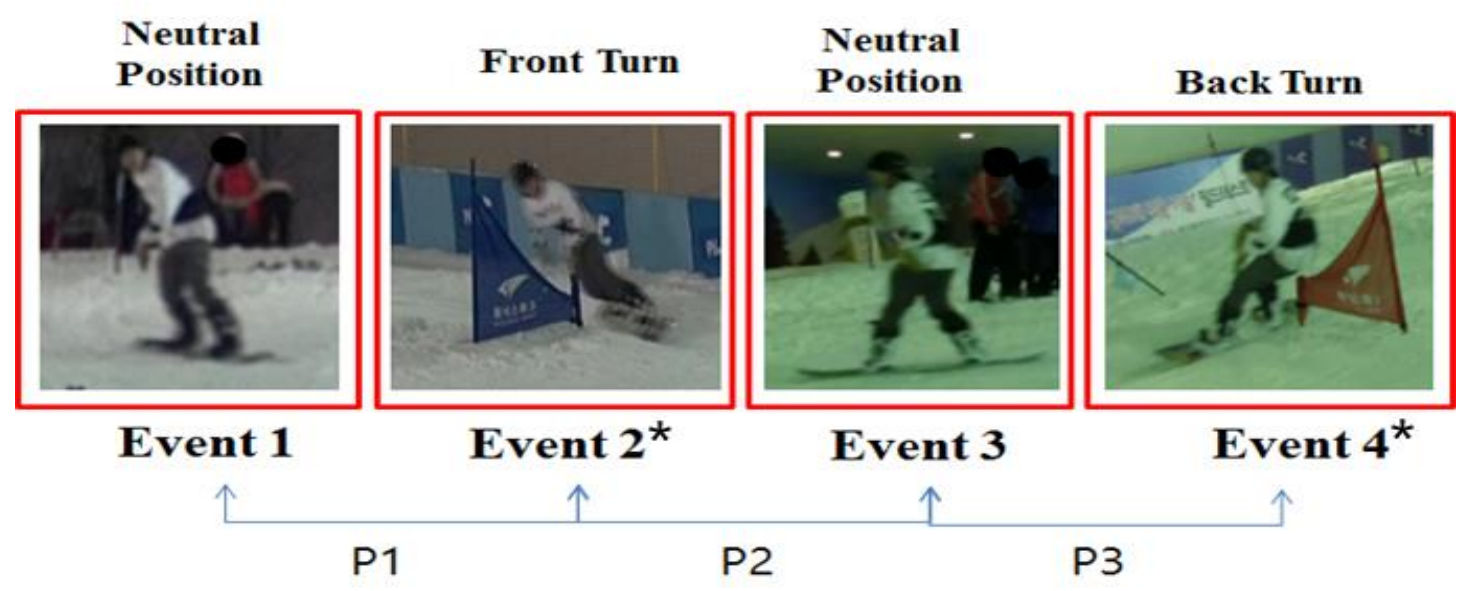

Figure 3. Event and Phase Setting

Snowboard riding is generally divided into three parts; front side turn (where the toe edge is used and the direction of progress can be watched), neutral position (where the center of gravity is not leaning toward any edge), and backside turn (where the toe edge is used and the direction of progress cannot be fully watched) and the starting direction of turns varies with situations. Event 2 and Event 4 were set to the moments when the center of gravity is lowest in the front side and backside turns respectively.

\section{2) Kinematic Variables}

Kinematic variables were calculated to see changes in the postures of the players during riding motions. The calculated kinematic variables are changes in the vertical height of the hip and changes in the lower limb joint angles.

\section{3) The EMG Variable}

Based on previous studies, EMG attachment regions were determined to be the lateral gastrocnemius muscle, the anterior tibial muscle, the biceps femoris muscle, the rectus femoris muscle, the vastus intermedius muscle, and the vastus lateralis muscle(Christophe Delecluse et al., 2001; Johnny Nilsson et al., 2004). The EMG raw data was full wave rectified to prevent the distortion of quantification by negative values and maintain all signal characteristics. In addition, a band pass filters $(20 \sim 500 \mathrm{~Hz})$ was applied to remove noise. Lastly, the rectified signals were integrated over a certain time interval to calculate the EMG integral using the following equation:

$$
i E M G=\int_{t}^{t+\tau}|E M G(t)| \cdot d t
$$

\section{4) Plantar Pressure Variable}

The foot area was generally divided into the forefoot, the mid foot, and the hind foot from the anatomical viewpoint to measure plantar pressure during snowboard riding motions and to collect related data. From the input data for the two feet, vertical forces, average pressure, and contact forces were calculated using the Pedar-X mobile expert version 7.3 software (Park Seung-Beom at al., 2007). The foot area division in the forefoot, the mid foot, and the hind foot for analysis of plantar pressure are as shown in Figure 4. 


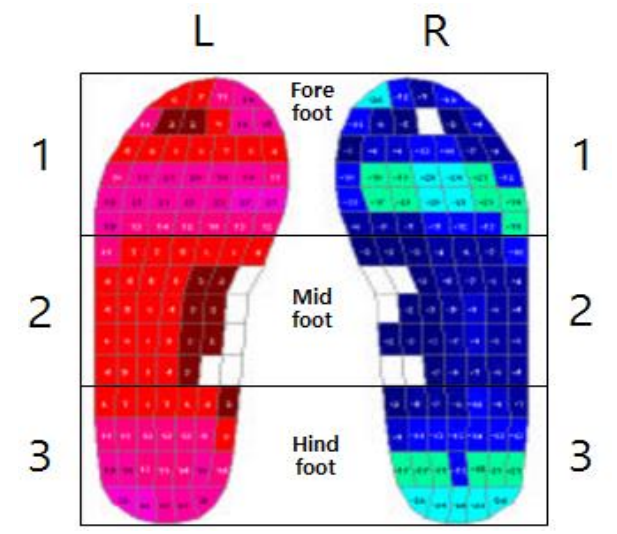

Figure 4. Foot Area Division

\section{Study Results and Discussion}

\subsection{Average Changes in the Z-axis of the Hip (Height \%)}

Table 3. Changes in the Vertical Height of the Hip

\begin{tabular}{c|l|c}
\hline Division & Event & B5 \\
\hline \multirow{4}{*}{ Rt.hip } & event 1 & $41.08 \pm 6.08$ \\
\cline { 2 - 3 } & event 2 & $33.75 \pm 5.74$ \\
\cline { 2 - 3 } & event 3 & $41.31 \pm 5.76$ \\
\cline { 2 - 3 } & event 4 & $24.90 \pm 4.29$ \\
\hline \multirow{4}{*}{ Lt.hip } & event 1 & $40.59 \pm 4.34$ \\
\cline { 2 - 3 } & event 2 & $30.78 \pm 4.02$ \\
\cline { 2 - 3 } & event 3 & $40.64 \pm 3.76$ \\
\cline { 2 - 3 } & event 4 & $26.23 \pm 2.80$ \\
\hline
\end{tabular}

Changes in the vertical height of the right and left hips can be interpreted as cases where the posture is heightened or lowered to move the center of gravity. The height of the right (approx. 41.8\%) and left hips (approx. 40.59\%) increased in the neutral position due to the 'up' motion to raise the posture before preparing for a turn(edge) in a certain direction. For the case of the right hip, the position becomes lower than the neutral position during both the front side (approx. 33.75\%) and backside (approx. 24.90\%) turns, with the 'down' motions taken during backside turns so that the height of the hip becomes lower than that during front side turns. This is also the case for the left hip. Care must be taken when the center of gravity has been excessively lowered as the center of gravity of the body may be moved away from the deck, leading to a decrease in the downhill speed due to the inertia moment. 


\subsection{Changes in Lower Limb Joint Angles}

Table 4. Changes in Lower Limb Joint Angles

\begin{tabular}{c|c|c|c|c}
\hline Division & e1 & e2 & e3 & e4 \\
\hline \hline R.ankle & $108.59 \pm 25.67$ & $96.52 \pm 25.30$ & $95.78 \pm 11.83$ & $113.21 \pm 4.21$ \\
\hline R.knee & $142.23 \pm 23.47$ & $123.88 \pm 5.27$ & $128.71 \pm 9.59$ & $122.81 \pm 17.25$ \\
\hline R.hip & $127.86 \pm 28.32$ & $122.33 \pm 37.09$ & $126.86 \pm 31.74$ & $114.10 \pm 31.51$ \\
\hline L.ankle & $117.01 \pm 14.46$ & $100.03 \pm 18.26$ & $106.10 \pm 5.05$ & $110.64 \pm 28.65$ \\
\hline L.knee & $138.33 \pm 9.02$ & $135.12 \pm 10.32$ & $145.51 \pm 9.17$ & $131.73 \pm 15.30$ \\
\hline L.hip & $116.27 \pm 15.21$ & $129.84 \pm 29.76$ & $127.83 \pm 17.40$ & $110.14 \pm 23.67$ \\
\hline
\end{tabular}

Among changes in lower limb joint angles, it can be seen that during the front side turns, the right ankle angle (approx. $96.5^{\circ}$ ) becomes smaller than the left ankle angle $\left(100^{\circ}\right)$ by approximately $4^{\circ}$ (dorsiflexion). This can be attributed to the fact that the right ankle is bent more than the left ankle such that the toe edge moves further into the snow. As with the ankle joints, it can be seen that the right knee joint angle (approx. $123^{\circ}$ )becomes smaller than the left knee joint angle (approx. $135^{\circ}$ ). In the principle of riding, the variation of knee joint flexion according to the variation of the angulation made through ankle joint angles can be said to be natural. The right hip joint angle (approx. $122^{\circ}$ ) becomes smaller than the left hip joint angle (approx. $129^{\circ}$ ) by approx. $7^{\circ}$ during front side turns and the left hip joint angle(approx. $110^{\circ}$ ) becomes smaller than the right hip joint angle(approx. $114^{\circ}$ ) by approx. $4^{\circ}$.

\subsection{Degree of Muscle Activation (EMG)}

P1 can be regarded to be the muscle activation section for front side turns and P3can be regarded to be the muscle activation section for backside turns. During the front side turns, the right vastus intermedius muscle $(13.92 \%)$, the right biceps femoris muscle (10.86\%), the right gastrocnemius muscle (13.99\%), and the right anterior tibial muscle $(17.17 \%)$ were shown to be major activated muscles. During backside turns, the right vastus lateralis muscle $(10.23 \%)$, the right vastus intermedius muscle $(15.95 \%)$, the left biceps femoris muscle $(10.04 \%)$, and the right anterior tibial muscle $(12.64 \%)$ were shown to be major activated muscles.

Table 5. Average EMG Values (uV) and Mobilization Rates (\%) of the Lower Limb Muscles by Phase

\begin{tabular}{|c|c|c|c|}
\hline Phase & Muscle & EMG(uV) & Mobilization rate(\%) \\
\hline \hline \multirow{4}{*}{ Phase 1 } & RT.RF & $30.05 \pm 16.05$ & $3.57 \pm 2.17$ \\
\cline { 2 - 4 } & LT.RF & $28.48 \pm 19.25$ & $3.52 \pm 2.86$ \\
\cline { 2 - 4 } & RT.VL & $65.45 \pm 30.37$ & $7.78 \pm 3.27$ \\
\cline { 2 - 4 } & LT.VL & $32.93 \pm 14.83$ & $4.12 \pm 2.51$ \\
\cline { 2 - 4 } & RT.VM & $146.18 \pm 166.71$ & $13.92 \pm 11.76$ \\
\cline { 2 - 4 } & LT.VM & $59.90 \pm 30.06$ & $6.93 \pm 4.14$ \\
\cline { 2 - 4 } & RT.BF & $102.78 \pm 76.52$ & $10.86 \pm 6.25$ \\
\hline
\end{tabular}




\begin{tabular}{|c|c|c|c|}
\hline & LT.BF & $74.20 \pm 93.52$ & $7.04 \pm 6.72$ \\
\hline & RT.GCM & $165.53 \pm 287.69$ & $13.39 \pm 20.25$ \\
\hline & LT.GCM & $27.44 \pm 17.52$ & $3.02 \pm 1.68$ \\
\hline & RT.TA & $132.50 \pm 10.15$ & $17.17 \pm 8.90$ \\
\hline & LT.TA & $71.70 \pm 48.57$ & $8.71 \pm 7.42$ \\
\hline \multirow{12}{*}{ Phase 2} & RT.RF & $24.06 \pm 11.23$ & $3.15 \pm 1.59$ \\
\hline & LT.RF & $26.10 \pm 13.07$ & $3.32 \pm 1.45$ \\
\hline & RT.VL & $55.48 \pm 7.37$ & $8.11 \pm 4.52$ \\
\hline & LT.VL & $39.05 \pm 16.64$ & $5.44 \pm 3.00$ \\
\hline & RT.VM & $201.20 \pm 278.44$ & $17.66 \pm 15.53$ \\
\hline & LT.VM & $77.33 \pm 32.88$ & $9.59 \pm 2.75$ \\
\hline & RT.BF & $70.08 \pm 50.84$ & $8.46 \pm 3.65$ \\
\hline & LT.BF & $73.48 \pm 98.38$ & $6.85 \pm 5.40$ \\
\hline & RT.GCM & $120.63 \pm 159.80$ & $13.76 \pm 16.42$ \\
\hline & LT.GCM & $31.32+21.59$ & $3.88 \pm 2.30$ \\
\hline & RT.TA & $75.10 \pm 25.24$ & $10.06 \pm 4.03$ \\
\hline & LT.TA & $75.10 \pm 57.04$ & $9.74 \pm 8.66$ \\
\hline Phase & Muscle & EMG (uV) & Mobilization rate $(\%)$ \\
\hline \multirow{12}{*}{ Phase 3} & RT.RF & $58.75 \pm 23.05$ & $5.03 \pm 2.93$ \\
\hline & LT.RF & $35.83 \pm 13.08$ & $2.98 \pm 1.14$ \\
\hline & RT.VL & $112.85 \pm 27.43$ & $10.23 \pm 5.46$ \\
\hline & LT.VL & $66.40 \pm 13.02$ & $6.17 \pm 3.40$ \\
\hline & RT.VM & $285.88 \pm 355.05$ & $15.95 \pm 11.13$ \\
\hline & LT.VM & $102.95 \pm 47.08$ & $8.14 \pm 2.50$ \\
\hline & RT.BF & $114.75 \pm 106.47$ & $7.04 \pm 2.65$ \\
\hline & LT.BF & $193.28 \pm 295.59$ & $10.04 \pm 10.43$ \\
\hline & RT.GCM & $136.65 \pm 201.48$ & $9.44 \pm 12.05$ \\
\hline & LT.GCM & $33.50 \pm 23.37$ & $2.32 \pm 0.82$ \\
\hline & RT.TA & $134.93 \pm 44.11$ & $12.64 \pm 7.44$ \\
\hline & LT.TA & $99.18 \pm 17.13$ & $10.07 \pm 3.71$ \\
\hline \multirow{4}{*}{ Phase 4} & RT.RF & $36.23 \pm 18.46$ & $3.66 \pm 1.42$ \\
\hline & LT.RF & $36.58 \pm 17.21$ & $3.96 \pm 1.54$ \\
\hline & RT.VL & $88.03 \pm 36.14$ & $10.23 \pm 6.52$ \\
\hline & LT.VL & $61.18 \pm 15.01$ & $8.54 \pm 6.28$ \\
\hline
\end{tabular}




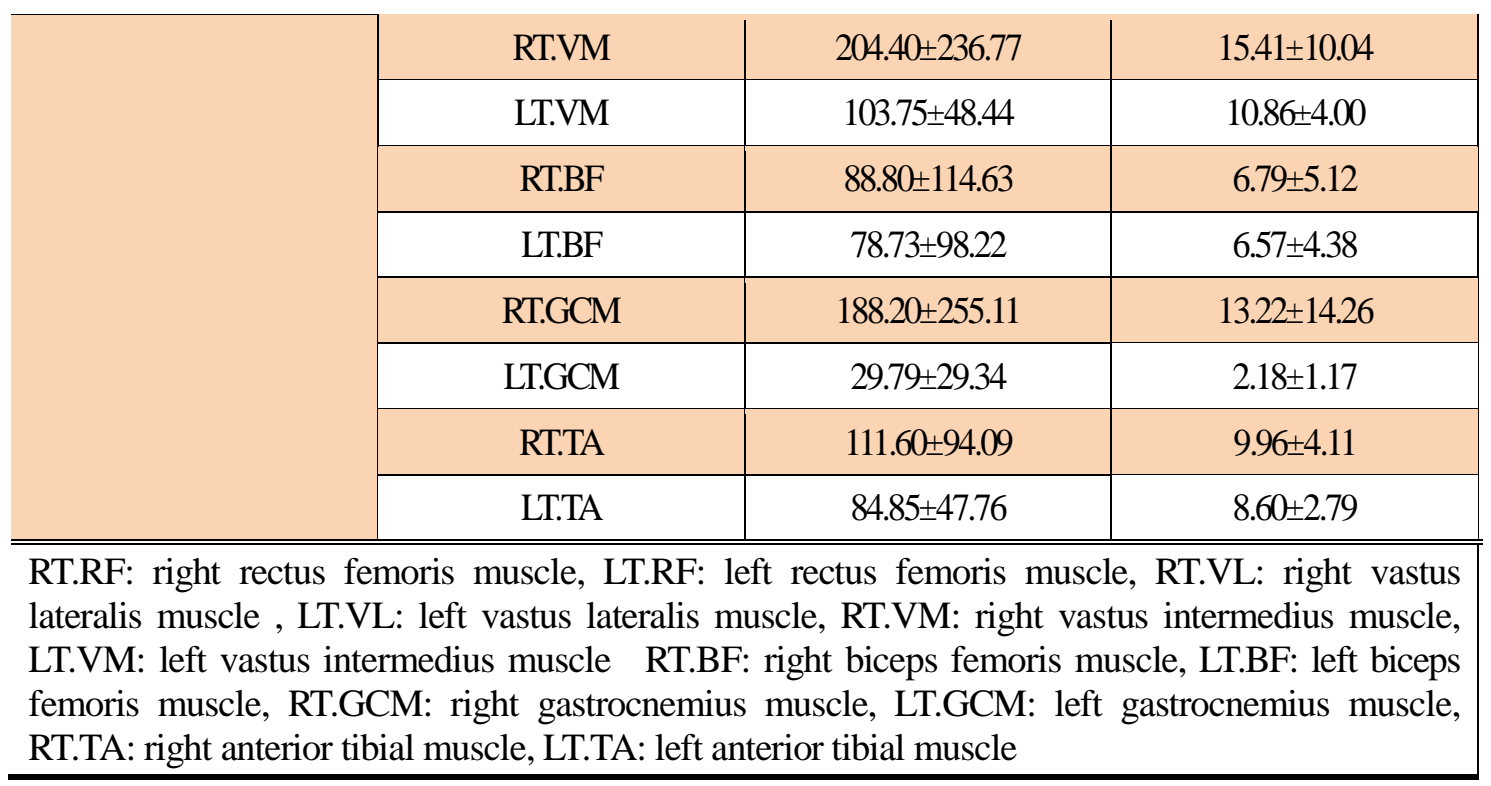

\subsection{Plantar Pressure Analysis}

E2 refers to the moment of front side turns and E4refers to the moment of backside turns. During the front side turns, the largest vertical force (approx. 213N) and 49\% of the plantar pressure act on the left front food. The largest vertical force (approx. 202N) and $54 \%$ of the plantar pressure act on the right hind foot. This may be due to the fact that although the center of gravity of the body was lower through the down motion, the neuromuscular responses cannot follow the right foot, where a large force of the toe edge acts on or is affected by the snow surface at the time of measurement. During the backside turns, the largest vertical force (approx. 222N) and 61\% of the plantar pressure act on the left hind foot and the largest vertical force (approx. 249N) and 70\% of the plantar pressure act on the right hind foot.

Table 6. Vertical Forces (N) and Averages (\%) of Plantar Pressure

\begin{tabular}{c|c|c|c}
\hline Event & Foot area & Verticalforce $(\mathbf{N})$ & Average pressure(\%) \\
\hline \hline \multirow{5}{*}{ Event 1 } & L1 & $107.55 \pm 215.09$ & $27.43 \pm 54.86$ \\
\cline { 2 - 4 } & L2 & $99.24 \pm 134.89$ & $18.21 \pm 25.42$ \\
\cline { 2 - 4 } & L3 & $198.96 \pm 157.16$ & $53.08 \pm 42.79$ \\
\cline { 2 - 4 } & R1 & $70.82 \pm 65.06$ & $16.46 \pm 15.09$ \\
\cline { 2 - 4 } & R2 & $115.01 \pm 52.37$ & $19.37 \pm 8.96$ \\
\cline { 2 - 4 } & R3 & $91.56 \pm 60.36$ & $25.42 \pm 16.24$ \\
\hline \multirow{6}{*}{ Event2 } & L1 & $213.15 \pm 158.68$ & $49.71 \pm 34.85$ \\
\cline { 2 - 4 } & L2 & $184.78 \pm 122.76$ & $33.02 \pm 24.53$ \\
& L3 & $73.05 \pm 85.26$ & $20.45 \pm 25.05$ \\
\cline { 2 - 4 } & R1 & $47.70 \pm 57.38$ & $11.38 \pm 14.69$ \\
& R2 & $147.58 \pm 44.77$ & $24.68 \pm 7.79$ \\
\hline
\end{tabular}




\begin{tabular}{c|c|c|c}
\hline \multirow{4}{*}{ Event } & L1 & $99.39 \pm 93.16$ & $23.32 \pm 22.08$ \\
\cline { 2 - 4 } & L2 & $153.15 \pm 80.64$ & $26.81 \pm 16.35$ \\
\cline { 2 - 4 } & L3 & $87.26 \pm 134.78$ & $22.17 \pm 32.63$ \\
\cline { 2 - 4 } & R1 & $153.60 \pm 64.87$ & $35.80 \pm 15.31$ \\
\cline { 2 - 4 } & R2 & $166.65 \pm 57.48$ & $28.96 \pm 12.97$ \\
\cline { 2 - 4 } & R3 & $52.06 \pm 33.57$ & $14.71 \pm 9.96$ \\
\cline { 2 - 4 } & L1 & $73.89 \pm 93.40$ & $15.64 \pm 19.08$ \\
\cline { 2 - 4 } & L2 & $126.08 \pm 69.34$ & $21.44 \pm 11.77$ \\
\cline { 2 - 4 } & L3 & $222.33 \pm 120.02$ & $61.70 \pm 34.41$ \\
\cline { 2 - 4 } & R1 & $81.06 \pm 142.24$ & $17.11 \pm 29.16$ \\
\cline { 2 - 4 } & R2 & $124.12 \pm 98.47$ & $20.65 \pm 14.93$ \\
\hline \hline
\end{tabular}

L1,L2,L3: left foot

$\mathrm{R} 1, \mathrm{R} 2, \mathrm{R} 3$ : rightfoot

\section{Conclusion and Proposal}

Based on the results of the present study, the following conclusions can be drawn. In neutral positions, the 'up' motions were taken in which the heights of the right and left hips were relatively the highest and at the moments of turns in both directions, 'down' motions were taken in which the heights of the right and left hips were relatively the lowest. During front side turns, the right ankle angle became smaller than the left ankle angle so that the toe edge moved deeper into the snow and the right knee joint was bent more than the left.

To review muscle activity during front side turns, the right vastus intermedius muscle, the right biceps femoris muscle, the right gastrocnemius muscle, and the right anterior tibial muscle were shown to be major activated muscles. During backside turns, the right vastus lateralis muscle, the right vastus intermedius muscle, the left biceps femoris muscle, and the right anterior tibial muscle $(12.64 \%)$ were observed as major activated muscles.

To review plantar pressure analysis during front side turns, the largest vertical force and plantar pressure acted on the left front food and the right hind foot. During backside turns, the largest vertical force and plantar pressure acted on the left hind foot and the right hind foot.

In further studies, the comparison of positions in successive turns is recommended.

\section{References}

[1] P. S. Beom and L. J. Suk, "Analysis of ground reaction force and plantar pressure according to the types of hiking boots and walking motions," Korean Journal of Sport Biomechanics, vol. 17, no. 4, (2007), pp. 190-200.

[2] C. Bladin, P. McCrory and A. Pogorzelski, "Snowboarding injuries: Current trends and fracture directions", Sports Medicine, vol. 34, (2004), pp. 133-139.

[3] C. Delecluse, T. Coeckelberghs and R. Vranken, "Knee angular displacement and extensor muscle activity in telemark skiing and in ski-specific strength exercises", University of San Francisco, Biomechanics Symposia, (2001).

[4] J. Nilsson and P. Haugen, Journal of Sports Sciences, vol. 22, (2004), pp. 357-364.

[5] M. Erich, "Comparisons of the ski turn techniques of experienced and intermediate skiers", Journal of Sports Sciences, vol. 16, no. 6, (1998), pp. 545-559. 
[6] P. Oshea, "Quantum Strength\& Power Training”, New York: Ballantine Books, (1998).

[7] M. L. Pollock and L. H. Wilmore, "Exercise in health and disease: Evaluation and prescription for prevention and rehabilitation, $2^{\text {nd }}$ ed", Philadelphia, Saunders, (1990).

[8] A. Pressman and D. H. Johnson, "A review of ski injuries resulting in combined injury to the anterior cruciate ligament and medial collateral ligaments", Arthroscopy, vol. 19, (2003), pp. 194-292.
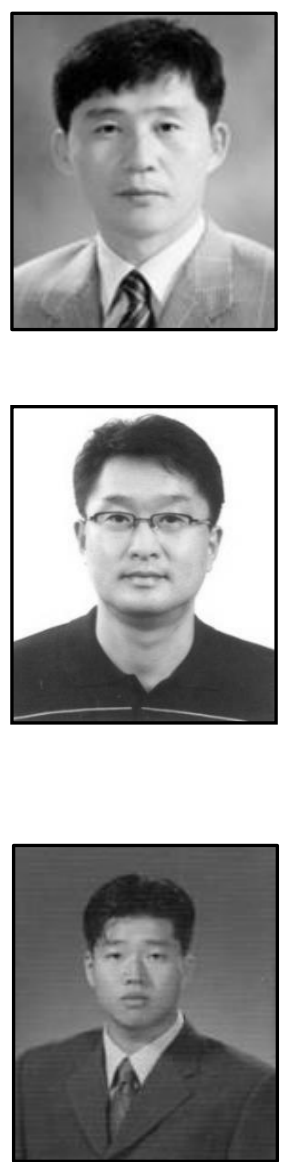

\section{Authors}

Chong-Hoon Lee, he received his BS, MS in Physical Education from Seoul National University. Doctor's degree from SungKyunKwan University (Shcool of sport science) in 1996. His major is a Sports Biomechanics. Professor, Dept. of Sports Sciences since 2000. Significant area of interest: Sport Biomechanical analysis, Development and verification of sports equipment. Korea Email: Leejh36@snut.ac.kr

Jin ho Back, he is a professor at Department of Leisure Sports, Kangwon National University Samcheok campus. He received his Ph.D. degree from Sungkyunkwan University in 1989, 1992 and 1997 respectively in Korea. He was a senior researcher at Korea institute of Sports science from 1997 to 2008. Currently, He is an executive director as well as editor of Korean Society of Sports Biomechanics (KSSB). His research interests include Sports Biomechanics and Motion Analysis. Korea Email: jhback@kangwon.ac.kr

Ki-Jeong Nam, he received his BS, MS and Ph.D in Physical Education from Seoul National University. His major is a Sports Biomechanics. He is now a researcher in the Sports Biomechanics $\mathrm{Lab}$ at the Seoul National University and the Seoul National University of Science \& Technology. department of Biomedical Engineering. His main research interests involve studying rehabilitation, injury and efficiency. 